\title{
LONGEST CYCLES IN CERTAIN BIPARTITE GRAPHS
}

\author{
PAK-KEN WONG \\ Department of Mathematics and Computer Science \\ Seton Hall University \\ South Orange, NJ 07079 USA
}

(Received July 10, 1995 and in revised form October 25, 1995)

\begin{abstract}
Let $G$ be a connected bipartite graph with bipartition $(X, Y)$ such that $|X| \geq|Y|(\geq 2), n$ $=|X|$ and $m=|Y|$ Suppose, for all vertices $x \in X$ and $y \in Y$, dist $(x, y)=3$ implies $d(x)+d(y) \geq n+1 \quad$ Then $G$ contains a cycle of length $2 m \quad$ In particular, if $m=n$, then $G$ is hamiltonian
\end{abstract}

KEY WORDS AND PHRASES: Bipartite graphs, 2-connected graphs, hamıltonıan graphs 1991 AMS SUBJECT CLASSIFICATION CODES: 05C38, $05 C 45$

\section{INTRODUCTION}

We consider only finite undirected graphs without loops or multıple edges. Our termınology is standard and can be found in [1]. Let $G=(V, E)$ be a graph For each vertex $x \in V$, let $D(x)=\{v \in V: v$ is adjacent to $x\}$. Then $d(x)=|D(x)|$ is the degree (valency) of $x$ in $G$

Let $G$ be a 2-connected graph. Suppose, for all vertices, $x, y \in V$, dist $(x, y)=2$ implies $\max \{d(x), d(y)\} \geq|V| / 2$ Then it was shown in [2] that $G$ is hamiltonian Some generalizations of this result can be found in [3]

The purpose of this paper is to obtain a similar result for bipartite graphs Let $G$ be a connected bipartite graph with bipartition $(X, Y)$ such that $|X| \geq|Y|(\geq 2), n=|X|$ and $m=|Y|$ If $m \neq n$, then $G$ cannot be hamiltonian However, $G$ may contain cycles Suppose, for all vertices $x \in X$ and $y \in Y$, dist $(x, y))=3$ implies $d(x)+d(y) \geq n+1$ Then we show that $G$ contains a cycle of length $2 m$ (Theorem 7) It is also shown that $G$ is 2-connected (Corollary 8)

As shown by an example in Section 3 , the condition "dist $(x, y)=3$ implies $d(x)+d(y) \geq n+1$ " cannot be replaced by a weaker condition "dist $(x, y)=3$ implies $\max (d(x), d(y)) \geq(n+1) / 2$ " Also this condition cannot be replaced by "dist $(x, y)=3$ implies $d(x)+d(y) \geq m+1$, if $m \neq n$

\section{RESULTS}

In this section, we assume that $G$ is a connected bipartite graph with bipartition $(X, Y)$ such that $|X| \geq|Y|(\geq 2)$ Let $n=|X|$ and $m=|Y|$ We also assume that, for all vertices $x \in X$ and $y \in Y$, dist $(x, y)=3$ implies $d(x)+d(y) \geq n+1$

If $S$ is a subgraph of $G$ and $v$ is a vertex of $S$, let $D_{S}(v)=\{u \in V(S): u$ is adjacent to $v\}$ and $d_{S}(v)=\left|D_{S}(v)\right|$ Let $P=\left\{w_{1}, w_{2}, \ldots, w_{2 r}\right\}$ be a longest path of length $2 r$ such that $w_{1}, w_{3}, \ldots, w_{2 r-1} \in X$ and $w_{2}, w_{4}, \ldots, w_{2 r} \in Y \quad \mathbf{A}$ path of even length is called an even path

LEMMA 1. The minimum degree of $G$ is at least two

PROOF. Suppose, on the contrary, there exists a vertex $x \in X$ such that $d(x)=1$ Since $G$ is connected, it is easy to see that there exists some $y$ in $G$ such that $\operatorname{dist}(x, y)=3$ and so $d(x)+d(y) \geq n+1 \quad$ Since $y$ is not adjacent to $x, d(y) \leq n-1 \quad$ But then $d(x)+d(y) \leq n$, which is impossible. Hence $d(x) \geq 2$ for all $x \in X$. Similarly, $d(y) \geq 2$ for all $y \in Y$ 
LEMMA 2. If $d_{P}\left(w_{1}\right)+d_{P}\left(w_{2 r}\right) \geq r+1$, then the vertices of $P$ form a cycle

PROOF. Suppose this is not true. Then $w_{1}$ is not adjacent to $w_{2 r}$ For each $w_{1} \in D_{P}\left(w_{1}\right)$ (with $i \neq 2$ ), we have $w_{2-1} \notin D_{P}\left(w_{2 r}\right)$, for otherwise $\left(w_{1}, w_{2}, w_{2+1}, \ldots, w_{2 r}, w_{2-1}, w_{2-2}, \ldots, w_{2}\right)$ is a cycle of length $2 r$ Since $w_{1} \notin D_{P}\left(w_{2 r}\right)$, we have

$$
\begin{gathered}
d_{P}\left(w_{2 r}\right) \leq(r-1)-\quad\left(d_{P}\left(w_{1}\right)-1\right) \\
\left(\text { take out } w_{1}\right) \quad(\text { take out } 2=2)
\end{gathered}
$$

Hence $d_{P}\left(w_{1}\right)+d_{P}\left(w_{2 r}\right) \leq r$, which is a contradiction.

LEMMA 3. If $d\left(w_{1}\right)+d\left(w_{2 \tau}\right) \geq n+1$, then the vertices of $P$ form a cycle.

PROOF. If $w_{1}$ is adjacent to $w_{2 r}$, the lemma is true. Hence we can assume that $w_{1}$ is not adjacent to $w_{2 r}$ By Lemma 2, it is sufficient to show that $d_{P}\left(w_{1}\right)+d_{P}\left(w_{2 r}\right) \geq r+1$ Since $P$ is a longest even path, either $d_{P}\left(w_{1}\right)=d\left(w_{1}\right)$ or $d_{P}\left(w_{2 r}\right)=d\left(w_{2 r}\right)$

CASE 1. Assume $d_{P}\left(w_{1}\right)=d\left(w_{1}\right)$ Then $d\left(w_{2 r}\right)-d_{P}\left(w_{2 r}\right) \leq n-r$ and so $d_{P}\left(w_{2 r}\right) \geq d\left(w_{2 r}\right)-n+r$ Hence $d_{P}\left(w_{1}\right)+d_{P}\left(w_{2 r}\right) \geq d\left(w_{1}\right)+d\left(w_{2 r}\right)-n+r \geq r+1$

CASE 2. Assume $d_{P}\left(w_{2 r}\right)=d\left(w_{2 r}\right)$ Then $d\left(w_{1}\right)-d_{P}\left(w_{1}\right) \leq m-r$ and so $d_{P}\left(w_{1}\right) \geq d\left(w_{1}\right)-m+r$ Hence $d_{P}\left(w_{1}\right)+d_{P}\left(w_{2 r}\right) \geq d\left(w_{1}\right)-m+r+d\left(w_{2 r}\right) \geq(n+1)-m+r=r+1+(n-m) \geq r+1$.

Therefore the lemma is true

In the following lemma, we assume that $d_{P}\left(w_{1}\right)=d\left(w_{1}\right)$ and $w_{1}$ is not adjacent to $w_{2 r}$ Since, by Lemma $1, d_{P}\left(w_{1}\right)=d\left(w_{1}\right) \geq 2$, there exists some $k(2<k<2 r)$ such that $w_{1}$ is adjacent to $w_{k}$ and $k$ is largest, this means, if $k^{\prime}>k$, then $w_{k^{\prime}}$, is not adjacent to $w_{1}$.

LEMMA 4. Suppose that $d_{P}\left(w_{1}\right)=d\left(w_{1}\right)$ and the vertices of $P$ do not form a cycle Then $d_{P}\left(w_{k+1}\right)+d_{P}\left(w_{2 r}\right) \leq r$

PROOF. By Lemmas 2 and $3, d\left(w_{1}\right)+d\left(w_{2 r}\right) \leq n$ and $d_{P}\left(w_{1}\right)+d_{P}\left(w_{2 r}\right) \leq r$. Hence $\operatorname{dist}\left(w_{1}, w_{2 r}\right)>3$ and so $k \neq 2 r-2$ Thus $k+2 \neq 2 r$. By the choice of $k, w_{k+2}$ is not adjacent to $w_{1}$ and so $\operatorname{dist}\left(w_{k+2}, w_{1}\right)=3$ which implies $d\left(w_{1}\right)+d\left(w_{k+2}\right) \geq n+1$ Hence by Lemma 3 , the vertices of $P$ cannot form a path of length $2 r$ with ends $w_{1}$ and $w_{k+2}$.

We claim that, for any $w_{\imath} \in D_{P}\left(w_{k+1}\right), w_{\imath-1} \notin D_{P}\left(w_{2 r}\right) \quad$ In fact, if $i=2$, then $w_{2 r}$ is not adjacent to $w_{1}$ If $2<i<k$ and $w_{\imath-1}$ is adjacent to $w_{2 r}$, then we have $w_{1}, w_{k}, w_{k-1}, \ldots, w_{\imath}, w_{k+1}, w_{k+2}, \ldots, w_{2 r}, w_{\imath-1}$, $w_{\imath-2}, \ldots, w_{2}$ This is a cycle of length $2 r$, which is impossible If $i=k$, then $w_{2 r}$ is not adjacent to $w_{k-1}$, because $\operatorname{dist}\left(w_{1}, w_{2 r}\right)>3$ If $i=k+2$, then $w_{2 r}$ is not adjacent to $w_{k+1}$, otherwise $\operatorname{dist}\left(w_{1}, w_{2 r}\right)=3$ If $k+4 \leq i \leq 2 r-2$ and $w_{2 r}$ is adjacent to $w_{\imath-1}$, then we have $w_{1}, w_{2}, \ldots, w_{k+1}, w_{\imath}, w_{\imath+1}, \ldots, w_{2 r}$, $w_{\imath-1}, w_{\imath-2}, \ldots, w_{k+2}$ This is a path of length $2 r$ with ends $w_{1}$ and $w_{k+2}$, which is impossible Therefore, for any $w_{\imath} \in D_{P}\left(w_{k+1}\right), w_{\imath-1} \notin D_{P}\left(w_{2 r}\right)$ and so $d_{P}\left(w_{2 r}\right) \leq r-d_{P}\left(w_{k+1}\right)$. Thus $d_{P}\left(w_{k+1}\right)+d_{P}\left(w_{2 r}\right) \leq r$

LEMMA 5. If $d_{P}\left(w_{1}\right)=d\left(w_{1}\right)$ and $d_{P}\left(w_{2 r}\right)=d\left(w_{2 r}\right)$, then the vertices of $P$ form a cycle

PROOF. Suppose, on the contrary, that the vertices of $P$ do not form a cycle Let $k$ be as in Lemma 4. From the proof of Lemma 4 , we have $\operatorname{dist}\left(w_{1}, w_{2 r}\right)>3$ and so $w_{k+1}$ is not adjacent to $w_{2 r}$ If $\operatorname{dist}\left(w_{k+1}, w_{2 r}\right)=3$, then $d\left(w_{k+1}\right)+d\left(w_{2 r}\right) \geq n+1$ Since $d\left(w_{2 r}\right)=d_{P}\left(w_{2 r}\right)$, it follows from the proof of Lemma 3 that $d_{P}\left(w_{k+1}\right)+d_{P}\left(w_{2 r}\right) \geq r+1$. But this contradicts Lemma 4. Thus dist $\left(w_{k+1}, w_{2 r}\right)>3$ If there exists some vertex $w_{\imath}$ which is adjacent to both $w_{k+2}$ and $w_{2 r}$, then $\operatorname{dist}\left(w_{k+1}, w_{2 r}\right)=3$, which is impossible Hence $w_{k+2}$ and $w_{2 r}$ cannot have a common neighbor on $P$ and so $d_{P}\left(w_{k+2}\right)+d_{P}\left(w_{2 r}\right) \leq r$ Therefore $d_{P}\left(w_{k+2}\right) \leq r-d_{P}\left(w_{2 r}\right)$. Since $\operatorname{dist}\left(w_{1}, w_{k+2}\right)=3$, we have $d\left(w_{1}\right)+d\left(w_{k+2}\right) \geq n+1$ Since $d\left(w_{1}\right)=d_{P}\left(w_{1}\right)$, by the proof of Lemma $3, d_{P}\left(w_{1}\right)+d_{P}\left(w_{k+2}\right) \geq r+1$. Hence, we have $r-d_{P}\left(w_{2 r}\right) \geq d_{P}\left(w_{k+2}\right) \geq r+1-d_{P}\left(w_{1}\right) \quad$ Therefore $d_{P}\left(w_{1}\right)-d_{P}\left(w_{2 r}\right) \geq 1$ and so $d\left(w_{1}\right)>d\left(w_{2 r}\right)$ By replacing $w_{2 r}$ with $w_{1}$ in the above argument, we can also show that $d\left(w_{2 r}\right)>d\left(w_{1}\right)$ This is impossible Hence the vertices of $P$ form a cycle.

LEMMA 6. There exists a cycle of length $2 r$ in $G$ 
PROOF. By Lemma 5, we can assume that, for each path $P$ of length $2 r$, either $d\left(w_{1}\right)>d_{P}\left(w_{1}\right)$ or $d\left(w_{2 r}\right)>d_{P}\left(w_{2 r}\right)$, otherwise the lemma is true. Let $P$ be a path of length $2 r$ with $d\left(w_{2 r}\right)>d_{P}\left(w_{2 r}\right) \quad$ (A similar argument holds for $d\left(w_{1}\right)>d_{P}\left(w_{1}\right)$.) Then, by the maximality of $P, d\left(w_{1}\right)=d_{P}\left(w_{1}\right)$ Since $d_{P}\left(w_{1}\right)=d\left(w_{1}\right) \geq 2$ (Lemma 1 ), there exists some $w_{k} \in P$ such that $w_{k}$ is adjacent to $w_{1}$ and $k \neq 2$ Also, we can assume that $k$ is the largest number among all such paths (with $d\left(w_{2 r}\right)>d_{P}\left(w_{2 r}\right)$ ) We claim that either $k=2 r$ or the vertices of $P$ form a cycle. Suppose this is not true If $k+2=2 r$, then $\operatorname{dist}\left(w_{1}, w_{2 r}\right)=3$ and so $d\left(w_{1}\right)+d\left(w_{2 r}\right) \geq n+1$ Hence by Lemma 3, the vertices of $P$ form a cycle, which is impossible. Thus $k+2 \neq 2 r$ and so $k \neq 2 r-2$. Since $k$ is the largest number, it follows that $k<2 r-2$ Hence $k+2<2 r$.

We claim that, if $w_{\imath} \in D_{P}\left(w_{1}\right)$ and $i \neq 2$, then $w_{\imath-1} \notin D_{p}\left(w_{k+2}\right)$ Suppose this is not true Since $k$ is the largest number, $4 \leq i \leq k$ and so $w_{\imath-1}, w_{\imath-2}, \ldots, w_{2}, w_{1}, w_{\imath}, w_{\imath+1}, \ldots, w_{2 r}$ is a path of length $2 r$ Since $d\left(w_{2 r}\right)>d_{P}\left(w_{2 r}\right)$, by the maximality of $P$, we have $d_{P}\left(w_{\imath-1}\right)=d\left(w_{\imath-1}\right)$ But $w_{\imath-1}$ is adjacent to $w_{k+2}$ Therefore $k$ is not the largest number among all such paths, which is a contraction Hence, if $w_{\imath} \in D_{P}\left(w_{1}\right)$ and $i \neq 2$, then $w_{\imath-1} \notin D_{P}\left(w_{k+2}\right)$. Since $w_{k+2}$ is not adjacent to $w_{1}$, we have

$$
d_{P}\left(w_{k+2}\right) \leq(r-1)-\left(d_{P}\left(w_{1}\right)-1\right)=r-d_{P}\left(w_{1}\right)
$$

Hence $d_{P}\left(w_{1}\right)+d_{P}\left(w_{k+2}\right) \leq r$. Since $\operatorname{dist}\left(w_{1}, w_{k+2}\right)=3$, we have $d\left(w_{1}\right)+d\left(w_{k+2}\right) \geq n+1$ Since $d_{P}\left(w_{1}\right)=d\left(w_{1}\right)$, the proof of Lemma 3 , we have $d_{P}\left(w_{1}\right)+d_{P}\left(w_{k+2}\right) \geq r+1$, which is a contraction Therefore either $k=2 r$, in which case we have a cycle, or the vertices of $P$ form a cycle of length $2 r$

We now have the main result of this paper.

THEOREM 7. Let $G$ be a connected bipartite graph with bipartition $(X, Y)$ such that $|X| \geq|Y|(\geq 2)$. Let $n=|X|$ and $m=|Y|$ Suppose, for all vertices $x \in X$ and $y \in Y$, dist $(x, y)=3$ implies $d(x)+d(y) \geq n+1$. Then $G$ contains a cycle of length $2 m$ In particular, if $m=n$, then $G$ is hamiltonian

PROOF. Let $P=\left(w_{1}, w_{2}, \ldots, w_{2 r}\right)$ be a longest even path in $G$ By Lemma 6, we can assume that $w_{1}$ is adjacent to $w_{2 r}$ We show that $r=m$. Suppose that this is not true Then $r<m$ and so $n \geq m \geq r+1$. Let $u \in X-P$ and $v \in Y-P$ Since $G$ is connected, there exists a shortest path $Q$ from $u$ to $P$ If $|Q|>1$, then there exists an even path with length greater than $2 r$ in $G$, which is impossible Hence $|Q|=1$ and so $d_{P}(u)=d(u) \geq 2$. Similarly $d_{P}(v)=d(v) \geq 2$ In particular, $u$ is not adjacent to $v$.

If there exists some $w_{\imath} \in P$ such that $w_{\imath}$ is adjacent to $u$ and $w_{\imath+1}$ (or $w_{\imath-1}$ ) is adjacent to $v$, then we have an even path of length greater than $2 r$, which is impossible. Therefore $d(u)+d(v) \leq r$ and $\operatorname{dist}(u, v)>3$ We can assume that $d(u) \geq d(v) \quad$ (A similar argument holds for $d(v) \geq d(u)$ ) Since $d(u) \geq 2$ and $d(u)+d(v) \leq r$, there exists some vertex, say $w_{3}$, such that $w_{3}$ is adjacent to $v$ and $w_{1}$ is not adjacent to $v \quad$ Since $\operatorname{dist}(u, v)>3, w_{2}$ is not adjacent to $u$ Since $\operatorname{dist}\left(w_{1}, v\right)=3$, $d\left(w_{1}\right)+d(v) \geq n+1$ Since $d(v)=d_{P}(v)$, by the proof of Lemma $3, d_{P}\left(w_{1}\right)+d_{P}(v) \geq r+1$ Hence

$$
d_{P}\left(w_{1}\right)+d_{P}(u) \geq d_{P}\left(w_{1}\right)+d_{P}(v) \geq r+1 \text {. }
$$

Thus there exists some vertex $w_{2} \in P$ such that $w_{2}$ is adjacent to both $u$ and $w_{1}$. It follows that $\operatorname{dist}\left(w_{2}, u\right)=3$ and so $d\left(w_{2}\right)+d(u) \geq n+1 \quad$ If $d\left(w_{2}\right)>d_{P}\left(w_{2}\right)$, then we clearly have an even path of length greater than $2 r$, because $v$ is adjacent to $w_{3}$. But this is impossible. Hence $d\left(w_{2}\right)=d_{P}\left(w_{2}\right)$ and so $d_{P}\left(w_{2}\right)+d_{P}(u)=d\left(w_{2}\right)+d(u) \geq n+1$. Since $n \geq r+1$, we have $d_{P}\left(w_{2}\right)+d_{P}(u) \geq r+2$ Therefore there exists some $k$ and $k^{\prime}$ with $k^{\prime}>k$ such that $w_{k}$ and $w_{k^{\prime}}$ are adjacent to $u$ and either $w_{k+1}$ is adjacent to $w_{2}$ or $w_{k^{\prime}-1}$ is adjacent to $w_{2}$, otherwise $d_{P}\left(w_{2}\right) \leq r-\left(d_{P}(u)-1\right)$ and so $d_{P}\left(w_{2}\right)+d_{P}(u) \leq r+1$ If $w_{k+1}$ is adjacent to $w_{2}$, then we have $w_{k^{\prime}+1}, w_{k^{\prime}+2}, \ldots, w_{1}, w_{2}, w_{k+1}, w_{k+2}$, $\ldots, w_{k^{\prime}}, u, w_{k}, w_{k-1}, \ldots, w_{3}, v$ and this is a path of length $2 r+2$, which is impossible. If $w_{k^{\prime}-1}$ is adjacent to $w_{2}$, then we have $w_{k+1}, w_{k+2}, \ldots, w_{k^{\prime}-1}, w_{2}, w_{1}, \ldots, w_{k^{\prime}}, u, w_{k}, w_{k-1}, \ldots, w_{3}, v$ and this is also a path of length $2 r+2$. This is a contraction Hence $r=m$ and this completes the proof of the theorem 
We have the last result of this section.

COROLLARY 8. $G$ is 2-connected

PROOF. By Theorem 7, $G$ contains a cycle $P$ of length $2 m$ If $m=n$, then $G$ is hamiltonian and so $G$ is 2 -connected Suppose $m<n$ For each vertex $x \in X-P$, we have $d_{P}(x)=d(x) \geq 2$ Hence, it follows that $G$ is also 2-connected.

\section{SOME REMARKS}

In this section, we give some remarks

REMARK 1. The condition "dist $(x, y)=3$ implies $d(x)+d(y) \geq n+1$ " in Theorem 7 cannot be replaced by a weaker condition $" \operatorname{dist}(x, y)=3$ implies $\max (d(x), d(y)) \geq(n+1) / 2$. In fact, let $G$ be the graph given in Figure 1, where the vertex partition is indicated by the filled and empty circles

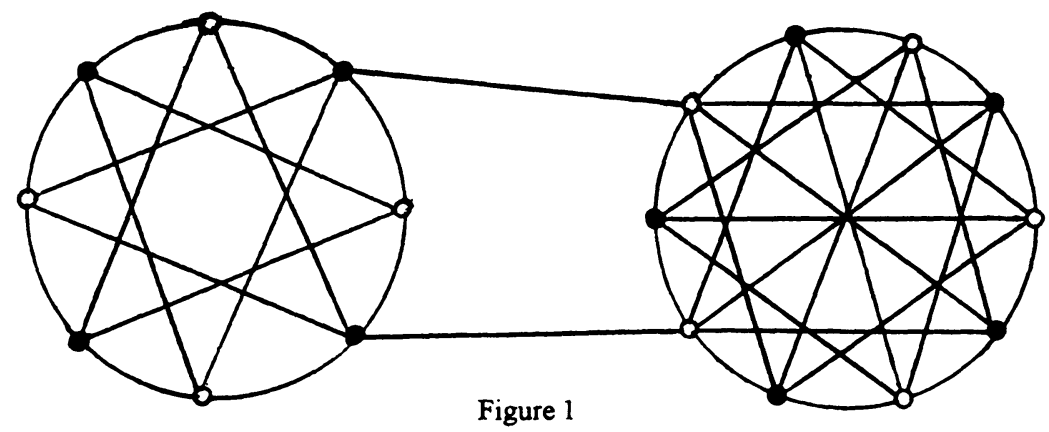

Then $n=m=9$ Clearly $G$ is not hamiltonian and $G$ satisfies the condition $\operatorname{dist}(x, y)=3$ implies $\max (d(x), d(y)) \geq(n+1) / 2$

REMARK 2. The condition "dist $(x, y)=3$ implies $d(x)+d(y) \geq n+1$ " in Theorem 7 cannot be replaced by the condition "dist $(x, y)=3$ implies $d(x)+d(y) \geq m+1$," if $m \neq n$ In fact, let $G$ be the graph given in Figure 2

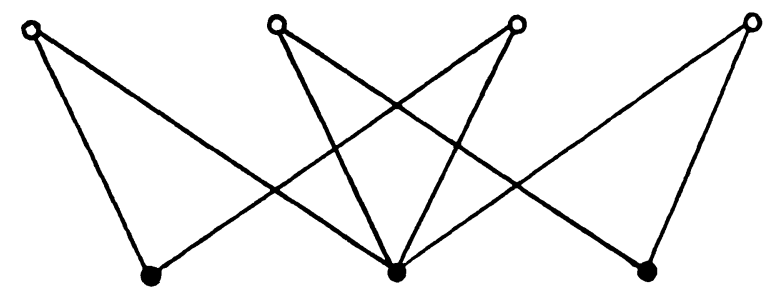

Figure 2

Then $n=4$ and $m=3$. Also $d(x)+d(y) \geq m+1=4$ for all $x \in X$ and $y \in Y$ But $G$ contains no cycle of length $2 m=6$. Since $G$ has a cut vertex, $G$ is not 2 -connected.

\section{REFERENCES}

[1] BONDY, J.A. and MURTY, U.S R., Graph Theory with Applications, MacMillan \& Co, London and Amer Elsevier, New York, 1976

[2] GAN, G H., New sufficient conditions for cycles in graphs, J. Combin. Theory, Ser B, 37 (1984), 221-227.

[3] GOULD, R.J., Updating the hamiltonian problem - a survey, J. Graph Theory, 15 (1991), 121-157 


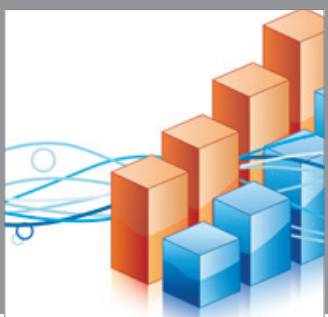

Advances in

Operations Research

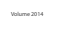

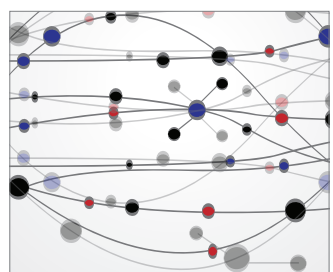

\section{The Scientific} World Journal
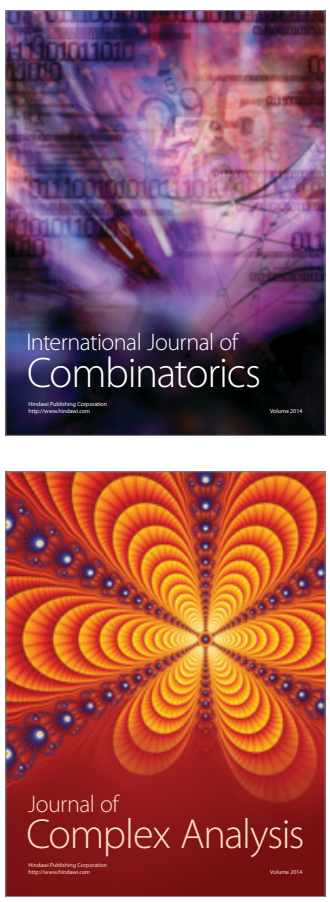

International Journal of

Mathematics and

Mathematical

Sciences
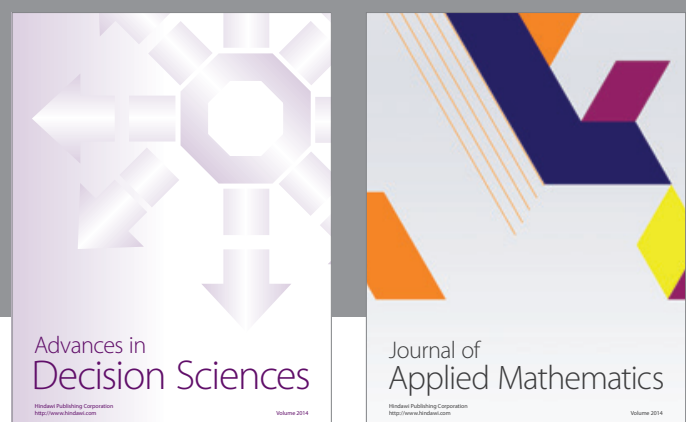

Journal of

Applied Mathematics
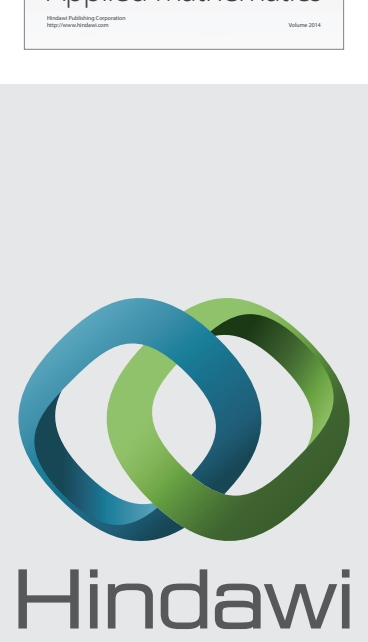

Submit your manuscripts at http://www.hindawi.com
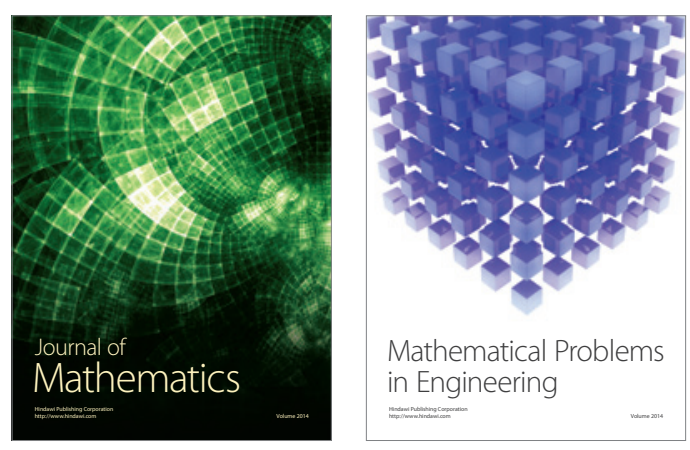

Mathematical Problems in Engineering
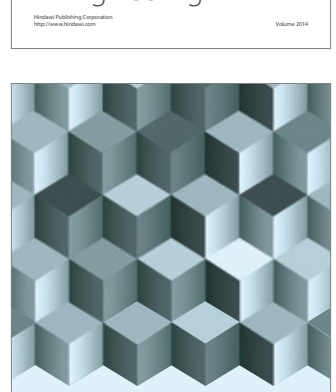

Journal of

Function Spaces
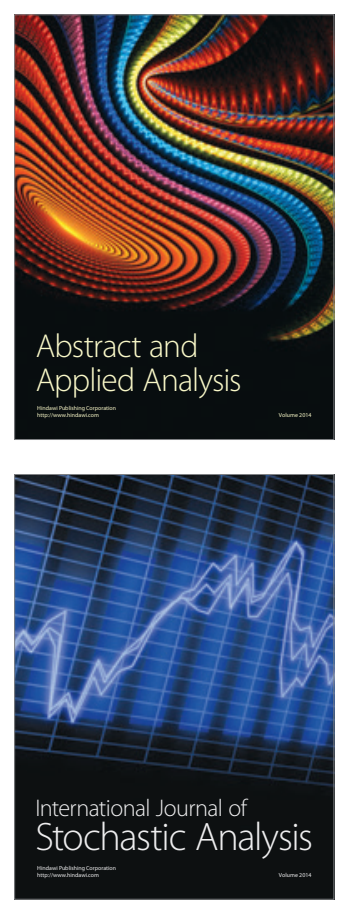

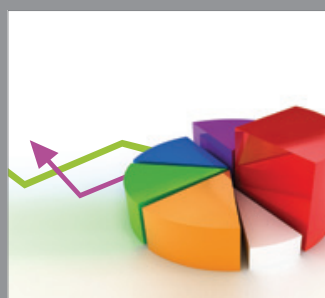

ournal of

Probability and Statistics

Promensencen
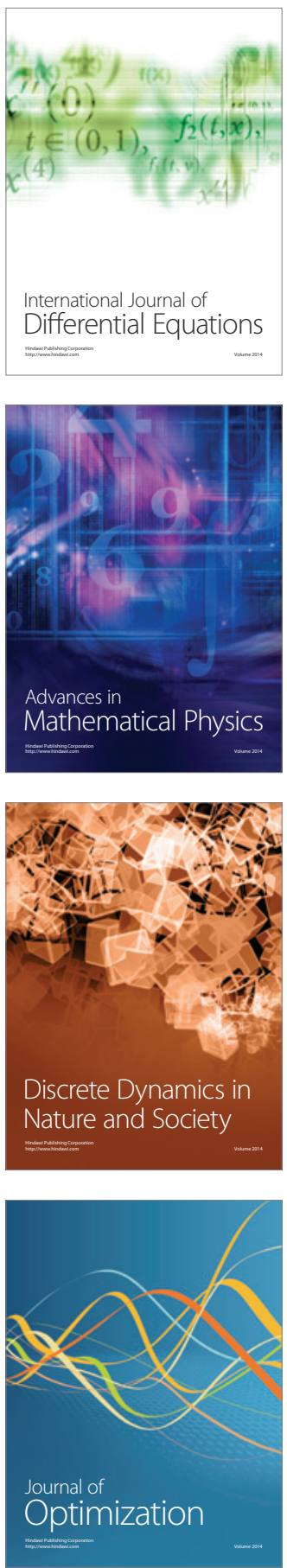